\title{
Stability Analysis of Tuberculosis Epidemic Model with Saturated Infection Force
}

\author{
Sutimin", Ratna Herdiana, R. Heri Soelistyo U, A. Henindya Permatasari \\ Department of Mathematics, Diponegoro University, Semarang, Indonesia
}

\begin{abstract}
A mathematical model with saturated infection rate is studied incorporating the effect of treatment for Tuberculosis (TB) infected individuals. The model is studied to analyze the local stability of equilibriums by center manifold. The effect of treatment, namely chemoprophylaxis for exposed individuals and therapy for infectious individuals is investigated numerically. The numerical results show that the chemoprophylaxis treatment is more effective in decreasing the spread of TB epidemic. Otherwise it increases the healthy individuals.
\end{abstract}

\section{Introduction}

Tuberculosis (TB) is a disease infecting lung and that is resulted in by the Mycobacterium Tuberculosis bacteria. As well as it attacks such as; the spine, kidney, brain, that can affect the central nervous system, lymphatic system, and kidneys. This bacterium can be prevented by immune system, but it will reactivate when it is weak [1].

The World Health Organization (WHO) has reported that TB remains becoming top infectious disease in the worldwide lead to mortality. Although, the death caused by TB decreased from 1.6 million in 2017 to 1.5 million in 2018 [2].

Mathematics has important role to analyze and describe the spread of tuberculosis epidemic through mathematical modeling. Currently, mathematical models have been applied to capture the dynamics of TB spread. The spread of TB epidemic model introducing the losing sight individuals, was studied by Bowong et al. [3] to analyze global behavior of the model. Other model of TB spread by consideration of treatments was also analyzed by [4]. By considering effect of drugs, the mathematical models were proposed and analyzed the stability of the model [5-8]. Egonmwan et al. [9-11] studied the model to analyze the stability of the model considering the effect of treatments.

We consider a dynamical model with treatments effect and saturated incidence rate. The dynamical behaviour of the model is discussed to study the local stability of equilibriums. The simulation results are provided to explore numerically the effect of treatments under the dynamical population in long terms.

\footnotetext{
* Corresponding author: sutimin.math.undip@gmail.com
} 


\section{Model Formulation}

The total population consists of five classes; susceptible $(S)$, exposed $(E)$, infected $(I)$, and recovery $(R)$ sub populations. The model can be given as follows,

$$
\begin{aligned}
& \frac{d S}{d t}=\lambda-\frac{\beta S I}{1+\alpha I}-\mu S+\omega R \\
& \frac{d E}{d t}=\frac{\beta S I}{1+\alpha I}+\gamma r_{2} I-(\phi+\mu) E \\
& \frac{d I}{d t}=\left(1-r_{1}\right) \phi E-\left[(\tau+\gamma) r_{2}+\mu+\delta\right] I \\
& \frac{d R}{d t}=\phi r_{1} E+\tau r_{2} I-(\mu+\omega) R
\end{aligned}
$$

We introduce two treatments, namely chemoprophylaxis for exposed individual with and therapy for infectious individuals with efficacies $r_{1}, r_{2}$, respectively. The recruitment rate of susceptible individuals is constant $\lambda$. The population is infected by infectious individual with the saturated incidence rate $\frac{\beta I}{1+\alpha I}$, where $\alpha$ is half-saturation constant. The natural death of individuals is $\mu$. Once chemoprophylaxis treatment, the fraction $\left(1-r_{1}\right) \phi$ of the exposed individuals move to infectious class, whereas other fraction $\phi r_{1} E$ becomes recovery individuals. Once therapy treatment, the fraction $\gamma r_{2}$ of infectious individuals return to the exposed class, whereas other fraction $\tau r_{2} I$ becomes recovery class. We assume that recovery individuals back to susceptible with the rate $\omega$.

\section{Model Analysis}

Now, we analyze the dynamical behaviour of the model related to the existence and local stability of equilibriums.

\subsection{Existence of equilibria}

The disease free equilibrium is $\mathrm{E}_{0}=\left(\frac{\lambda}{\mu}, 0,0,0\right)$, and the basic reproduction ratio can be defined as (see [12]),

$$
\mathfrak{R}_{0}=\frac{\lambda\left(1-r_{1}\right) \phi \beta}{\mu\left(r_{2} \gamma\left(\mu+\phi+r_{1} \phi\right)+(\phi+\mu)\left(\tau r_{2}+\delta+\mu\right)\right)}
$$

The endemic state (1) is given by,

$$
\bar{S}=\frac{r(\mu+\omega)(\alpha \mu \bar{I}+\beta \bar{I}+\mu)}{(\alpha \bar{I}+1)\left[\lambda r(\mu+\omega)+\omega \bar{I}\left(\gamma r_{1} r_{2}+\delta r_{1}+\mu r_{1}+\tau r_{2}\right]\right.}, \bar{E}=\frac{\left(\gamma r_{2}+\tau r_{2}+\delta+\mu\right) \bar{I}}{r \phi}
$$


$\bar{R}=\frac{\tau r_{2} \bar{I}\left(r+r_{1}\right)}{r(\mu+\omega)}+\frac{r_{1} \bar{I}\left(\gamma r_{2}+\delta+\mu\right)}{r(\mu+\omega)}$, where $r=1-r_{1}$, and $\bar{I}$ fulfils the linear polynomial of $b_{1} \bar{I}+a_{0}=0$, where

$$
\begin{aligned}
a_{1}= & (\delta+\mu)[\mu \alpha(\mu+2 \phi)(\mu+\omega)+\beta \phi(\omega r+\mu)]+\mu r_{2}(2 \alpha(\mu+\omega)+\beta)\left(\gamma r_{1}+\tau\right) \phi \\
& +\alpha \mu^{2} r_{2}(\mu+\omega)(\tau+\gamma)>0 \\
a_{0}= & \mu(\mu+\omega)\left[\gamma r_{2}\left(\phi r_{1}+\mu\right)+(\phi+\mu)\left(\tau r_{2}+\delta+\mu\right)\right]\left(1-\mathfrak{R}_{0}\right) .
\end{aligned}
$$

The condition $\mathfrak{R}_{0}>1$ guarantees that the endemic state exists and unique.

\subsection{Local Stability of Equilibriums}

We analyze the local stability of $\mathrm{E}_{0}=\left(\frac{\lambda}{\mu}, 0,0,0\right)$ that is given in the next Theorem 1 .

Theorem 1. If $\mathfrak{R}_{0}<1$, the equilibrium point $\mathrm{E}_{0}$ is locally stable.

Proof: Linearization of the model (1) in matrix can be given by

$$
J\left(E_{0}\right)=\left(\begin{array}{cccc}
-\mu & 0 & -\frac{\beta \lambda}{\mu} & -\omega \\
0 & -\mu-\phi & \frac{\beta \lambda}{\mu}+\gamma r_{2} & 0 \\
0 & \phi r & -(\tau+\gamma) r_{2}-\mu-\delta & 0 \\
0 & \phi r_{1} & \tau r_{2} & -\mu-\omega
\end{array}\right)
$$

The eigen values of the matrix (7) are $\chi_{1}=-\mu, \chi_{2}=-\mu-\omega$ and others fulfill the polynomial, $a_{2} \chi^{2}+a_{1} \chi+a_{0}=0$, where $a_{2}=\mu, a_{1}=\mu\left(\gamma r_{2}+\tau r_{2}+\delta+2 \mu+\phi\right)$, and $a_{0}=\mu r_{2}\left(\phi r_{1}+\mu\right)+\mu(\phi+\mu)\left(\tau r_{2}+\delta+\mu\right)\left(1-\mathfrak{R}_{0}\right) . \quad$ It is seen that roots of this polynomial are negative if $\mathfrak{R}_{0}<1$. It completes the proof.

Next, we verify the local dynamic for endemic equilibrium provided in Theorem 2.

Theorem 2: If $\mathfrak{R}_{0}>1$, then $\mathrm{E}^{*}=(\bar{S}, \bar{E}, \bar{I}, \bar{R})$ is locally stable.

Proof: We use center manifold to prove the local stability. The bifurcation parameter $\beta$ is taken by determining $\mathfrak{R}_{0}=1$, and we have $\beta^{*}=\frac{\mu(\mu+\phi)\left[\delta+\mu+(\gamma+\tau) r_{2}\right]}{\lambda \phi r}-\frac{\mu \gamma r_{2}}{\lambda}$. Inserting $\beta^{*}$ into the matrix $J\left(E_{0}\right)$ is obtained, 


$$
J\left(E_{0}, \beta^{*}\right)=\left(\begin{array}{cccc}
-\mu & 0 & \gamma r_{2}-\frac{(\mu+\phi)\left(\gamma r_{2}+\tau r_{2}+\delta+\mu\right)}{\phi r} & -\omega \\
0 & -\mu-\phi & -\gamma r_{2}+\frac{(\mu+\phi)\left(\gamma r_{2}+\tau r_{2}+\delta+\mu\right)}{\phi r} & 0 \\
0 & \phi r & -(\tau+\gamma) r_{2}-\mu-\delta & 0 \\
0 & \phi r_{1} & \tau r_{2} & -\mu-\omega
\end{array}\right)
$$

The eigen values of the above matrix $J\left(E_{0}, \beta^{*}\right)$ are $\chi_{1}=-\mu, \chi_{2}=-\mu-\omega, \chi_{3}=0$, and $\chi_{4}=-\left(\gamma r_{2}+\tau r_{2}+\delta+2 \mu+\phi\right)$. The matrix $J\left(E_{0}, \beta^{*}\right)$ has one simple eigen value zero, and if the right eigen vector corresponding to eigen value $\chi_{3}=0$ is $\vec{W}=\left(w_{1}, w_{2}, w_{3}, w_{4}\right)^{\mathrm{T}}$ , then it holds $J\left(E_{0}, \beta^{*}\right) \cdot \vec{W}=0$, it is obtained, $w_{2}=\frac{\left(\gamma r_{2}+\tau r_{2}+\delta+\mu\right) r_{1} w_{3}}{r(\mu+\omega)}+\frac{\tau r_{2} w_{3}}{\mu+\omega}$ $w_{1}=-r r_{2}(\gamma \mu+\gamma \omega+\omega \tau)+(\mu+\omega r)\left(\gamma r_{2}+\tau r_{2}+\delta+\mu\right) \quad, w_{3}=w_{3}, \quad$ and $\quad w_{4}=\frac{\tau r_{2} w_{3}}{\mu+\omega}+$ $\frac{\left(\gamma r_{2}+\tau r_{2}+\delta+\mu\right) w_{3}}{r(\mu+\omega)}$. Let $\vec{V}=\left(v_{1}, v_{2}, v_{3}, v_{4}\right)$ to be the left eigen vector corresponding to $\chi_{3}=0$, then $\vec{V} \cdot J\left(E_{0}, \beta^{*}\right)=0$. So, we obtain $\vec{V}=\left(0, v_{2}, \frac{\mu+\phi}{\phi r} v_{2}, 0\right)$. The relation of $w_{3}, v_{2}$, is founded by solving $\vec{W} \cdot \vec{V}=1$, and it is obtained $v_{2}=\frac{\phi r}{w_{3}\left(\gamma r_{2}+\tau r_{2}+\delta+2 \mu+\phi\right)}, w_{3}=w_{3}>0$. Next, the model (1) can be represented in the notation $f_{1}, f_{2}, f_{3}, f_{4}$ for $\frac{d S}{d t}, \frac{d E}{d t}, \frac{d I}{d t}, \frac{d R}{d t}$, respectively. Using centre manifold theorem, it is founded

$$
\begin{aligned}
a=2 v_{2} w_{1} w_{3} \frac{\partial^{2} f_{2}}{\partial S \partial I}=-\frac{2 v_{2} \beta r_{2} w_{3}^{2}\left[\mu\left(\gamma r_{1}+\tau\right) \phi+\mu(\tau+\gamma)(\mu+\omega)\right]}{\mu(\mu+\omega)} \\
-\frac{2 v_{2} \beta w_{3}^{2}\left[(\mu+\delta)\left(\omega \phi r+\mu^{2}+\mu \omega+\mu \phi\right]\right.}{\mu \phi r(\mu+\omega)}<0 \text {, and } b=\frac{\lambda v_{2} w_{3}}{\mu}>0
\end{aligned}
$$

Thus, it follows from centre manifold shows that $\mathrm{E}^{*}$ is locally stable, if $\mathfrak{R}_{0}>1$.

\section{Simulation Results}

In the simulations, we investigate the analytical results to describe the dynamical of the spread of TB epidemic in long terms. The values of parameter and sources are available in Table 1. 
Table 1. Parameter values and sources.

\begin{tabular}{|c|c|c|}
\hline Parameters & Values & Sources \\
\hline$\alpha$ & $(0.1-1.25)$ & {$[13]$} \\
\hline$\lambda$ & 0.08 & {$[5]$} \\
\hline$\beta$ & 0.04 & {$[13]$} \\
\hline$\phi$ & 0.005 & {$[3]$} \\
\hline$\tau$ & 0.02 & {$[3]$} \\
\hline$\gamma$ & 0.01 & Estimate \\
\hline$r_{1}$ & 0.9706 & {$[14]$} \\
\hline$r_{2}$ & 0.8182 & {$[3]$} \\
\hline$\mu$ & 0.0101 & {$[3]$} \\
\hline$\delta$ & 0.02272 & {$[3]$} \\
\hline$\omega$ & 0.3 & {$[15]$} \\
\hline
\end{tabular}

We simulate the effect of chemoprophylaxis treatment and therapy in preventing the spread of TB epidemic. The simulation are given in the following figures.
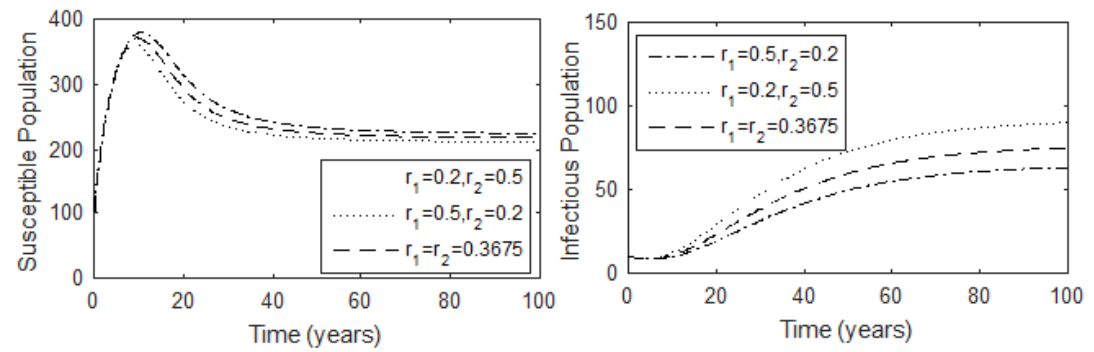

Fig.1. The effect of treatments under the dynamics of susceptible and infectious population in different efficacies.

We consider the scenarios of treatments when chemoprophylaxis and therapy are administered simultaneously. In the simulation, we determine overall efficacy $\bar{\varepsilon}=1-\left(1-r_{1}\right)\left(1-r_{2}\right)=0.6$. The first scenario, when we choose $r_{1}=0.5$ and $r_{2}=0.2$. The second scenario is for $r_{1}=0.2$ and $r_{2}=0.5$, and the third scenario for $r_{1}=r_{2}=0.3675$. In Figure 1, shows that chemoprophylaxis treatment is more effective in increasing the susceptible individuals compared to therapy. On the other hand, this treatment is faster in decreasing the infectious population compared to therapy. It may show that chemoprophylaxis treatment is effective to overcome the spread of TB epidemic. 


\section{Conclusion}

A dynamical model of TB epidemic with saturated infection rate with treatment was studied. The model was analyzed to explore the local stability of equilibrium states. The local stability of equilibriums was analysed using linearization and centre manifold. The local stability of disease free equilibrium is fulfilled if $\mathfrak{R}_{0}<1$ and otherwise local stability of endemic equilibrium is achieved if $\mathfrak{R}_{0}>1$.

In numerical simulations, by using the efficacy values of chemoprophylaxis treatment and therapy, we may conclude that chemoprophylaxis treatment may be more effective in preventing the spread of TB epidemic compared to therapy.

\section{Acknowledgement}

This work is supported by NON-APBN FSM 2020, Diponegoro University, Semarang, Indonesia No: 1963/UN7.5.8/PP/2020.

\section{References}

1. M.M. Dago, M.O Ibrahim, A..S. Tosin, Int. J. Adv. Sci. Eng. Tech. 3 (2015).

2. The World Health Organization, Global Tuberculosis Report 2019.

3. S. Bowong and J. J. Tewa, Commun. Nonlin. Sci. Numer. Simul. 14 (2009).

4. B.K. Mishra, J. Srivastava, J. Egypt. Math. Soc. 22, ( 2014).

5. H.F. Huo, M.X. Zou, Appl. Math. Model. 40, (2016).

6. S. Elkhaiar, A. Kaddar, Res. Appl. Math., 1 (2017).

7. Y. Yu, Y. Shi, W. Yao, Infect. Dis. Model., 3, ( 2018).

8. M. Ronoh, R. Jaroudi, P. Fotso, V. Kamdoum, N. Matendechere, J. Wairimu, R. Auma, J. Lugoye, App. Math. 7 (2016).

9. A.O. Egonmwan, D. Okuonghae, J. Appl. Math. Comput. (2018).

10. L.N. Nkamba, T.T. Manga, F. Agouanet, M.L.M. Manyombe, J. Biol. Dyn. 13,1(2019).

11. H. Guo, M.Y. Li, Canad. Appl.Math. Quart. 14 (2006).

12. O. Diekmann, J.A.P. Heesterbeek, 5, JohnWiley \& Sons, west sussex, (2000).

13. J. Zhang, J. Jia, and X. Song, Sci. World J., (2014).

14. S. Kim, A. A. de los Reyes, and E. Jung, J. Theor. Biol., (2018).

15. B.K. Mishra, J. Srivastrava, J. Egyp. Math. Soc. 22, (2014). 